\title{
NEUROPROTECTIVE EFFECT OF CAJANINSTILBENE ACID AGAINST CEREBRAL ISCHEMIA AND REPERFUSION DAMAGES BY ACTIVATING AMPK/NRF2 PATHWAY
}

\author{
Hui $\mathrm{Xu}^{1}$, Jiangang Shen ${ }^{1}$, Jianbo Xiao ${ }^{2}$, Feng $\mathrm{Chen}^{3}$, and Mingfu Wang ${ }^{1}$ \\ ${ }^{1}$ University of Hong Kong \\ ${ }^{2}$ Jinan University \\ ${ }^{3}$ Shenzhen University
}

April 28, 2020

\begin{abstract}
BACKGROUND AND PURPOSE Neuroprotection is an important strategy for ischemic stroke treatment. Cajaninstilbene acid (CSA), a unique stilbenoid with a styryl group, is a potential neuroprotective agent. Hence, this study aimed to evaluate the neuroprotective effect and molecular mechanism of CSA against cerebral ischemia/reperfusion (I/R) damages. EXPERIMENTAL APPROACH The ischemic stroke was modeled by oxygen and glucose deprivation/reoxygenation (OGD/R) in SH-SY5Y cells and transient middle cerebral artery occlusion/reperfusion $(\mathrm{MCAO} / \mathrm{R})$ in rats. Tert-butyl hydroperoxide (t-BHP) was used to induce oxidative stress in cells. The signaling pathways were analyzed by Western blotting and inhibitor blocking. KEY RESULTS CSA possessed significant neuroprotective activity, as evidenced by the reduced cell death in OGD/R or tBHP injured cells, and decreased infarct volume and neurological deficits in MCAO/R rats. Further studies indicated that the protective effect was achieved via the antioxidant activity of CSA, which decreased the oxidative stress and its related mitochondrial dysfunction in cells. Notably, Nrf2 was activated in cells and MCAO/R rats by CSA, and the inhibition of Nrf2 by brusatol weakened CSA-mediated neuroprotection. Furthermore, after applying a series of kinase inhibitors, CSA-induced Nrf2 activation was markedly inhibited by BML-275 (an AMPK inhibitor), implying that AMPK was the dominant kinase to regulate the Nrf2 pathway for CSA's neuroprotective effects with enhanced AMPK phosphorylation observed both in vivo and in vitro. CONCLUSION AND IMPLICATION CSA exerted neuroprotection via activating AMPK/Nrf2 pathway to reduce I/R-induced cellular oxidative stress and mitochondrial disfunction. CSA could be a potential neuroprotective drug candidate for the treatment of ischemic stroke.
\end{abstract}

\section{Bullet points summary:}

What is already known

1. CSA had neuroprotective effect in corticosterone damaged PC12 cells.

2. CSA possessed a styryl group, a potential neuroprotective group for ischemic stroke.

What this study adds

1. CSA exerted neuroprotection in OGD/R cell model and MCAO/R rat model with low effective dosage.

2. The pharmacological mechanism of CSA involved the activation of AMPK/Nrf2 mediated antioxidation.

Clinical significance 
CSA might be a potential drug candidate/leading compound for acute ischemic stroke treatment.

\section{Introduction}

Neuroprotection is the approach to protect neurons from different pathological factors under several abnormal cerebral conditions, such as Parkinson's diseases, ischemic stroke and traumatic brain injury. Neuroprotective agents can be administrated before the onset of these neurological diseases to reduce the related risk factors or after the onset of diseases to prevent the spreading of injuries from neurons to neighboring ones (Chang $\&$ Ho, 2019). Notably, the development of neuroprotective agents for ischemic stroke has drawn much attention in recent years. Ischemic stroke is caused by the occlusion of cerebral arteries, leading to the deprivation of glucose and oxygen to the blocked brain tissue, which then causes a series of damaging events to neurons including energy depletion, intracellular $\mathrm{Ca}^{2+}$ accumulation, excitotoxicity, free radical release and inflammation (Kunz, Dirnagl \& Mergenthaler, 2010). To tangle with this ischemic condition is to achieve reperfusion via thrombolytic therapy, mainly intravenous injection of recombination tissue plasminogen activator (r-tPA), as soon as possible. However, reperfusion would further promote free radical release and inflammation, causing secondary injuries to neurons (Doyle, Simon \& Stenzel-Poore, 2008). Hence, neuroprotection is necessary for ischemic stroke patients, and many events or molecular targets can be modulated to produce potential protective effects within the cascade of cerebral ischemia and reperfusion (I/R).

Oxidative stress is one of the most important neuroprotective targets. The free radicals generated during cerebral $\mathrm{I} / \mathrm{R}$ can directly attack brain tissues, triggering mitochondrial dysfunction, inflammation and apoptosis of neurons. Furthermore, free radicals can enhance the permeability of blood-brain barrier via promoting the activation of matrix metalloproteases (MMPs), increasing the risks of cerebral hemorrhage and brain edema (Allen \& Bayraktutan, 2009). Many studies have focused on evaluating the neuroprotective potential of antioxidants, and one of the most famous agents is edaravone, which is found to reduce cerebral $\mathrm{I} / \mathrm{R}$ induced brain damages in different ischemic stroke models due to its free radical scavenging activity (Watanabe, Tanaka, Yuki, Hirai \& Yamamoto, 2018). Notably, edaravone has been officially approved for the treatment of ischemic stroke in 2001 and amyotrophic lateral sclerosis (ALS) in 2017 (Simion \& Jurcau, 2019).

Nuclear factor erythroid 2 related factor 2 (Nrf2) is an important transcriptional factor to regulate the gene expression of diverse cytoprotective proteins, particularly in the antioxidant systems. Once activated, Nrf2 translocates to the nucleus and activates the expression of various antioxidant enzymes, including $\mathrm{NAD}(\mathrm{P}) \mathrm{H}$ quinone oxidoreductase 1 (NQO1) and heme oxygenase-1 (HO-1) (de Vries et al., 2008). Since Nrf2 is fundamental to the cellular redox homeostasis, some natural Nrf2 activators including sulforaphane and tert-butylhydroquinone have shown remarkable efficacy in different animal models of ischemic stroke (Jiang et al., 2017). Nrf2 activation is regulated by multiple kinases, such as the mitogen-activated protein kinases (MAPKs), phosphatidylinositol 3-kinase/protein kinase B (PI3K/AKT) and AMP-activated protein kinase (AMPK). These kinases are reported to promote the Nrf2 translocation to nucleus via regulation of Keap1 or directly phosphorylation on Nrf2 (Joo, Kim, Lee, Kim, Koo \& Kim, 2016; Qi et al., 2017). Recently, AMPK mediated Nrf2 activation has been regarded as a critical neuroprotective target. As an example, Wu et al. reported that neferine, a natural alkaloid, could promote Nrf2 activation to protect cerebral I/R injured mitochondria in neurons (Wu, Chen, Yang, Duan, Li \& Chen, 2019). Another study suggested that HP-1c, a novel derivative of dl-3-n-butyphthalide, activated the AMPK/Nrf2 pathway to suppress the oxidative stress and inflammation in a transient focal cerebral ischemia model (Wang et al., 2018).

Pigeon pea (Cajanus cajan (L.)) is traditionally considered to have medical potential, and has been applied to the treatment of various diseases, such as diabetes, osteoporosis and necrosis of femoral head (Syed \& $\mathrm{Wu}, 2018$ ). One of the bioactive components in its leaves is cajaninstilbene acid (3-hydroxy-4-prenyl-5methoxystilbene-2-carboxylic acid, CSA), a stilbenoid composed of a styryl group and the scaffold of aspirin. CSA has recently drawn much attention due to its broad range of bioactivities, including anti-viral, anti- 
inflammatory, anti-oxidative, anti-bacterial, and cytoprotective effects, as well as treatment of osteonecrosis of femoral head and postmenopausal osteoporosis (Ji et al., 2016; Sun et al., 2019). Recently, we were focusing on screening neuroprotective agents for ischemic stroke from natural stilbenoids and flavonoids, and have found that some natural products that possessed a styryl group exerted stronger activities. Besides, some preliminary data showed that CSA prevented corticosterone-induced mitochondrial apoptosis in PC12 cells (Jiang et al., 2014). Furthermore, CSA was found to activate the Nrf2/ARE pathway in HepG2 cells at very low concentration (Liang et al., 2013). Based on those facts, it is reasonable to hypothesize that CSA might possess the neuroprotective activity against cerebral I/R injuries. Hence, the aim of this study is to investigate the neuroprotective effect of CSA in cerebral I/R injuries and its underlying protective mechanisms.

\section{Materials and methods}

CSA preparation

CSA (purity of 98\%) was extracted from the leaves of pigeon pea in our lab. The chemical structure of CSA was identified by chromatographic and spectral analysis and shown in Fig. 1 (Huang et al., 2016).

\subsection{Cell line, cell models and drug treatment}

SH-SY5Y neuroblastoma cells was obtained from American Type Culture Collection (ATCC, VA, USA). They were cultured at $37^{\circ} \mathrm{C}$ with the high glucose DMEM medium (Gibco, Gaithersburg, USA) supplemented with 10\% FBS (Gibco, Gaithersburg, USA) and 1\% penicillin-streptomycin (Gibco, Gaithersburg, USA) in a humidified atmosphere of $5 \% \mathrm{CO}_{2}$. Two cell models were used in this study: oxygen and glucose deprivation/reoxygenation (OGD/R) model and tert-butyl hydroperoxide (t-BHP) induced oxidative stress model. OGD/R model was established according to Feng et al. (Feng et al., 2018). Briefly, cells were washed with PBS (Sigma, St. Louis, USA), and then cultured with no glucose DMEM medium (Gibco, Gaithersburg, USA) in a hypoxia chamber (Billups-Rothenberg, Delmar, USA) perfused with $95 \% \mathrm{~N}_{2}$ and $5 \% \mathrm{CO}_{2}$ for $8 \mathrm{~h}$ at $37^{\circ} \mathrm{C}$. After that, cells were cultured at the normal cultural conditions for another $16 \mathrm{~h}$ to achieve reoxygenation. t-BHP-induced oxidative stress model was performed by treating cells with $100 \mu \mathrm{M}$ of t-BHP (Sigma, St. Louis, USA). Cells were pretreated with CSA for $24 \mathrm{~h}$ before they were subjected to OGD/R or t-BHP damages. The inhibitors, including SB203580, SP600125, U0126, BML-275 and brusatol (Sigma, St. Louis, USA), were added along with CSA if needed.

\subsection{Cell viability and LDH release}

Cell viability was measured with the Cell Counting Kit-8 (CCK-8, Dojindo Laboratories, Kumamoto, Japan). Cells were incubated with the CCK-8 solution in DMEM medium (1:10) for $1.5 \mathrm{~h}$, and then absorbance was measured at $450 \mathrm{~nm}$. LDH release was evaluated via detecting the LDH level in the cultural supernatant with a Pierce LDH Cytotoxicity Assay kit (Thermo Fisher Scientific, Waltham, USA).

\subsection{Mitochondrial membrane potential $\left(\Delta \Psi_{\mathrm{m}}\right)$}

JC-1 probe was used to detect the $\Delta \Psi_{\mathrm{m}}$ of SH-SY5Y cells. Briefly, cells were stained with $10 \mu \mathrm{g} / \mathrm{mL}$ JC-1 (Beyotime Institute of Biochemistry, Shanghai, China) at $37^{\circ} \mathrm{C}$ in the dark for $30 \mathrm{~min}$ and washed with PBS. Fluorescence was analyzed with the flow cytometer and cells with a higher ratio of green signals to red signals was considered to possess the decreased $\Delta \Psi_{\mathrm{m}}$.

\subsection{Cytochrome c release}

Cytochrome c release was detected with the Cytochrome c Releasing Apoptosis Assay kit (Abcam, Cambridge, UK) by detecting the cytochrome c content in cytoplasm. Briefly, cells were collected and homogenized with a Dounce tissue grinder. The supernatant of the homogenate was collected as the cytosolic fraction. Cytochrome c content was then analyzed by the standard Western blotting procedure with the monoclonal mouse anti-cytochrome $c$ antibody. $\beta$-actin was used as the internal control.

$2.6 \mathrm{Na}^{+}-\mathrm{K}^{+}$ATPase and $\mathrm{Ca}^{2+}-\mathrm{Mg}^{2+}$ ATPase activity 
The ATPase activities were measure with the Minim ATP Enzyme Test kit (Nanjing Jiancheng bioengineering institute, Nanjing, China) via measuring the inorganic phosphorus produced by enzymatic ATP hydrolysis. Briefly, cells were collected, homogenized, and then $\mathrm{Na}^{+}-\mathrm{K}^{+}$ATPase and $\mathrm{Ca}^{2+}-\mathrm{Mg}^{2+}$ ATPase activities in the homogenate were measured, respectively. One unit of ATPase was defined as the amount of enzyme that generated $1 \mu \mathrm{M}$ inorganic phosphate in $1 \mathrm{~h}$ at $37^{\circ} \mathrm{C}$. Protein content of the homogenate was measured with bicinchoninic acid (BCA) method to normalize the results.

\subsection{Intracellular reactive oxygen species (ROS) detection}

Intracellular ROS was measured with the DCFH-DA fluorescent probe. Cells were incubated with $10 \mu \mathrm{M}$ DCFH-DA (Sigma, St. Louis, USA) in the dark for $40 \mathrm{~min}$ at $37{ }^{\circ} \mathrm{C}$ and washed three times with PBS. Fluorescence was then analyzed by the flow cytometer. The ROS contents were expressed as the mean fluorescence intensity of stimulated samples versus that of control.

2.8 Superoxidase dismutase (SOD) activity and malondialdehyde (MDA) content

Intracellular SOD and MDA levels were measured with the SOD and MDA detecting kits (Nanjing Jiancheng bioengineering institute, Nanjing, China), respectively. Briefly, cells were collected, homogenized, and then centrifuged to get the supernatant. SOD was measured with the WST-1 method, and one unit of SOD was defined as the amount of SOD that inhibited $50 \%$ of the WST-1 formazan production. MDA was detected with the TBA (thiobarbituric acid) method. Protein content of supernatant was measured using the BCA method to normalize the results.

\subsection{Animal model and drug treatment}

The transient intraluminal suture middle cerebral artery occlusion (tMCAO) rat model was used in this research. The use of animals in this study was approved by the Committee on the Use of Live Animals in Teaching and Research, The University of Hong Kong (CULATR 4765-18). Adult male Sprague Dawley rats (250-280 g) were supplied by the Laboratory Animal Unit, The University of Hong Kong. Rats were raised at $22 \pm 2{ }^{\circ} \mathrm{C}$ with $12 \mathrm{~h}$ light and $12 \mathrm{~h}$ dark. The $\mathrm{MCAO} / \mathrm{R}$ model was conducted according to Feng et al. (Feng et al., 2018). Briefly, rats were firstly anesthetized with $4 \%$ isoflurane (Abbott, USA), and then maintained with $2 \%$ isoflurane during the whole operation process. The left common carotid artery (CCA), external carotid artery (ECA), and internal carotid artery (ICA) were exposed, and nerves around the arteries were carefully dissected. After that, a stump was made on ECA to insert a monofilament coated with silicon on the tip (0.36 mm, Jialing Co. Ltd., China). The monofilament was advanced from ECA to ICA and then to the root of the middle cerebral artery (MCA) to achieve cerebral ischemia. After ischemia for $2 \mathrm{~h}$, the monofilament was removed and the blood flow to MCA was restored. The sham control rats underwent the same operation of MCAO rats, but no monofilament was inserted to block the MCA. Rats were randomly divided into 5 groups ( $\mathrm{n}=10$ per group): sham control, $\mathrm{MCAO} / \mathrm{R}$ control, $2.5 \mathrm{mg} / \mathrm{kg} \mathrm{CSA}, 5 \mathrm{mg} / \mathrm{kg} \mathrm{CSA}$, and $5 \mathrm{mg} / \mathrm{kg}$ CSA $+2 \mathrm{mg} / \mathrm{kg}$ brusatol groups. CSA was dissolved in the mixture of ethanol (45\%), PEG400 (25\%), and saline (30\%). Drugs were intraperitoneally (i.p.) injected immediately after reperfusion. The sham control and $\mathrm{MCAO} / \mathrm{R}$ control rats were intraperitoneally injected with the same volume of the mixture solution (45\% ethanol, 25\% PEG400 and 30\% saline).

\subsection{Neurological scores and infarct volume}

Neurological scores were obtained by testing rats with the modified neurological severity score (mNSS) scale at $24 \mathrm{~h}$ after reperfusion. The mNSS scale ranges from 1 to 18 points, including motor, sensory, and reflex tests. A higher score indicates more severe neurological damages (Mao et al., 2013). Infarct volume was evaluated with TTC staining. Briefly, after reperfusion for $24 \mathrm{~h}$, rats were sacrificed through cardiac perfusion with PBS. Brain tissues were then collected, sliced into $2 \mathrm{~mm}$ coronal section and stained with $2 \%$ TTC solution at $37^{\circ} \mathrm{C}$ for $20 \mathrm{~min}$. The stained slices were photographed and analyzed with Image J. Infarct volume was calculated as (right hemisphere size - red part size of left hemisphere) / (right hemisphere size $\times 2) \times 100 \%$.

2.11 Nuclear Nrf2 translocation 
Nuclear Nrf2 translocation was evaluated by measuring Nrf2 content in nucleus. Cytoplasmic and nuclear fraction were separated with the NE-PER Nuclear and Cytoplasmic Extraction Reagents (Thermo Fisher Scientific, Waltham, USA). Nrf2 content in the nuclear extract was further tested by the standard Western blot procedure. Histone H3 (HH3) was used as the internal control. Data were analyzed with the image J software.

\subsection{Western blotting}

Cells or brain tissues were lysed with RIPA buffer (Thermo Fisher Scientific, Waltham, USA) containing 1 mM PMSF (Sigma, St. Louis, USA) and phosphate inhibitor cocktails (Sigma, St. Louis, USA), and total proteins were collected. SDS-PAGE was used to separate the proteins $(10 \mu \mathrm{g})$, and separated proteins were then transferred to the polyvinylidene fluoride (PVDF) membrane (Bio-rad, Hercules, USA). The membrane was blocked with $5 \%$ nonfat milk or BSA (bovine serum albumin, Sigma, St. Louis, USA) and incubated with the corresponding primary antibodies overnight at $4{ }^{\circ} \mathrm{C}$. After being washed with TBST, the membrane was then incubated with horseradish peroxidase-conjugated anti-mouse (Santa Cruz Biotechnology, Dallas, USA, 1:2000) or anti-rabbit (Abcam, Cambridge, UK, 1:5000) secondary antibodies for $1 \mathrm{~h}$ at room temperature. Protein bands were visualized with an enhanced chemiluminescence (ECL) substrate kit (Bio-rad, Hercules, USA) and analyzed with the Image J software. Primary antibodies used in this research were as follows. Antibodies from Abcam (Cambridge, UK): Cytochrome c (ab110325, 1:250), Nrf2 (ab31163, 1:1000) and HO-1 (ab13243, 1:2000); Antibodies from Cell signaling technology (Danvers, USA): NQO1 (62262, 1:1000), Keap1 (8047, 1:1000), p-Erk (4370, 1:2000), Erk (9102, 1:2000), p-p38 MAPK (4511, 1:1000), p38 MAPK (8690, 1:1000), p-JNK (9255, 1:1000), JNK (9252, 1:1000), p-AKT (9271, 1:1000), AKT (9272, 1:1000), p-AMPK (2535, 1:1000), AMPK (5831, 1:1000), HH3 (4499, 1:2000) and $\beta$-actin (3700, 1:5000).

\subsection{Statistical analysis}

All data were presented as mean \pm standard deviation (SD) of at least three independent experiments using the SPSS statistics 22 (IBM). Differences between the means of the individual groups were assessed by oneway analysis of variance (ANOVA) followed by the Tukey's test. Differences were considered significant at $P<0.05$.

\section{Results}

\subsection{CSA showed neuroprotection both in vitro and in vivo}

OGD/R model was widely used to mimic the cerebral I/R conditionsin vitro, so the neuroprotective effect of CSA was firstly evaluated in SH-SY5Y cells with OGD/R model. As shown in Fig. 2A, CSA enhanced the cell viability that was decreased by OGD/R damages, indicating its protective effects against OGD/Rinduced injuries. Oxidative stress played critical role in cerebral I/R induced damages. Fig. 2 also indicated that CSA reduced cell death induced by t-BHP (a ROS inducer), as evidenced by the increased cell viability (Fig. 2B) and reduced LDH release (Fig. 2C). Hence, the protective effect of CSA might be exerted at least partially through reducing the intracellular oxidative stress and its related injuries. In vivo evaluation was done with the $\mathrm{tMCAO} / \mathrm{R}$ rat model. Intraperitoneal injection of CSA was found to reduce the infarct volume and neurological deficits in $\mathrm{MCAO} / \mathrm{R}$ rats (Fig. 2D-F). In summary, CSA showed neuroprotection against cerebral I/R and oxidative stress induced injuries.

\subsection{CSA protected SH-SY5Y cells from OGD/R and t-BHP induced oxidative stress}

As mentioned above, CSA might attenuate the oxidative stress induced damages, so the antioxidant ability of CSA was further evaluated. Fig. 3 showed that the DCFH-DA fluorescent intensity was lower in CSA-treated cells than that in t-BHP $(0.1-1 \mu \mathrm{M})$ or OGD/R $(0.1-0.5 \mu \mathrm{M})$ injured cells, that is, CSA significantly reduced the intracellular ROS level induced by t-BHP and OGD/R damages. Furthermore, MDA was a biomarker of the oxidative stress by reflecting the degree of membrane lipid peroxidation. It was found that both t-BHP and OGD/R damages increased the MDA level in SH-SY5Y cells. However, SH-SY5Y cells showed decreased MDA content after treated with CSA by $33.30 \%$ in t-BHP model and $22.12 \%$ in OGD/R model $(0.5 \mu \mathrm{M}$ of CSA, Fig. 3). SOD was the first line to defense against the ROS (Alscher, Erturk \& Heath, 
2002). It was found that SOD activity was also significantly enhanced by CSA treatment (0.1 or $0.5 \mu \mathrm{M})$ when compared to t-BHP or OGD/R groups (Fig. 3). Those data implied that CSA reduced the oxidative stress and enhanced the antioxidant ability of SH-SY5Y cells.

\subsection{CSA alleviated the mitochondrial dysfunction in SH-SY5Y cells}

Mitochondria are the main target of oxidative stress and release more free radicals after damaged. Improved mitochondria function would be observed under reduced oxidative stress condition (Yang, Mukda \& Chen, 2018). To further evaluate the antioxidant and mitochondrial protective activity of CSA, mitochondria function was detected via measuring mitochondrial membrane potential $\left(\Delta \Psi_{\mathrm{m}}\right)$, cytochrome $\mathrm{c}$ release and ATPase activity. JC-1 fluorescence probes, which emitted red signals at normal $\Delta \Psi_{\mathrm{m}}$ and green signals at decreased $\Delta \Psi_{\mathrm{m}}$, were used to detect the mitochondrial membrane potential. $\Delta \Psi_{\mathrm{m}}$, a biomarker of the mitochondrial function, was decreased under cerebral I/R damages (Sanderson, Reynolds, Kumar, Przyklenk \& Hüttemann, 2013). As shown in Fig. 4A-C, cells damaged by t-BHP showed high percentage of depolarized cells (cells with decreased $\Delta \Psi_{\mathrm{m}}$ ) and low ratio of red/green signal, implying that t-BHP decreased the $\Delta \Psi_{\mathrm{m}}$ of SH-SY5Y cells. After treated with CSA, cells possessed improved $\Delta \Psi_{\mathrm{m}}$, as evidenced by the decreased percentage of depolarized cells and increased ratio of red/green signal compared to t-BHP treated cells. Mitochondrial dysfunction would lead to the release of many pro-apoptotic factors, such as cytochrome c (Andrabi, Ali, Tabassum, Parveen \& Parvez, 2019). Fig. 4D indicated that pretreatment of cells with CSA markedly decreased the cytochrome c release, as the cytosolic cytochrome c level of CSA-treated cells was much lower than that of t-BHP treated cells (decreased by $44.17 \%$ at $0.5 \mu \mathrm{M}$ ). $\mathrm{Na}^{+}-\mathrm{K}^{+}$ATPase (NKA, sodium pump) and $\mathrm{Ca}^{2+}-\mathrm{Mg}^{2+}$ ATPase (CMA, calcium pump) could sense the changes of oxygen availability and adjust their activity to the ATP production rates, that is, they could reflect the intracellular ATP level and mitochondrial energy metabolism function (Bogdanova, Petrushanko, Hernansanz-Agustín \& MartínezRuiz, 2016). It was found that CSA $(0.5 \mu \mathrm{M})$ enhanced the NKA and CMA activities that were suppressed by t-BHP (Fig. 4E-F). Further studies with OGD/R model also observed the up-regulated NKA and CMA activities by CSA $(0.5 \mu \mathrm{M})$ treatment (Fig. 4G-H). It could conclude from the above data that CSA improved the mitochondrial function of t-BHP or OGD/R injured cells, and this protective effect was achieved at least partially through reducing the oxidative stress in CSA treated cells.

\subsection{CSA promoted Nrf2 activation in both SH-SY5Y cells and MCAO/R rats}

Nrf2 was vital for the systemic antioxidant defense system. To explore the molecular targets that CSA activated to exert its antioxidant activity, key factors of Nrf2 pathway were detected. Nrf2 was kept in the cytoplasm by binding to the Kelch-like ECH-associated protein 1 (Keap1) at normal condition. Under stimulated condition, it was separated from Keap1 and translocated into the nucleus, binding to the ARE (antioxidant responsive element) promoter regions and activating its target genes, such as HO-1 and NQO1 (Liu et al., 2017). As shown in Fig. 5A-F, the protein levels of total-Nrf2, nuclear-Nrf2, HO-1 and NQO1 were up-regulated in SH-SY5Y cells after treated with CSA for $24 \mathrm{~h}$, implying that CSA promoted the activation of Nrf2 pathway. Generally, the effective concentrations of CSA for Nrf2 pathway in SH-SY5Y cells were found to be between $0.01-1 \mu \mathrm{M}$, with the best activity observed at 0.1 or $0.5 \mu \mathrm{M}$. The attenuated activity at 1 to $5 \mu \mathrm{M}$ might due to the increased cytotoxicity of CSA in SH-SY5Y cells. Furthermore, CSA also increased the protein levels of total-Nrf2, nuclear-Nrf2, HO-1 and NQO1 in t-BHP injured cells (Fig. 5). The nuclear-Nrf2 content of CSA $(0.5 \mu \mathrm{M})$ treated cells was $27.87 \%$ higher than that of t-BHP injured cells (Fig. 5L). Keap1 was down-regulated in CSA treated cells, which was consistent with the enhanced Nrf2 nuclear translocation of CSA (Fig. 5K). Next, the Nrf2 activation was tested in MCAO/R rats. As shown in Fig. 6, promoted Nrf2 nuclear translocation, up-regulated HO-1 and NQO1 expression, and suppressed Keap1 level were also observed in CSA treated MCAO/R rats. Intraperitoneal injection of CSA (5 mg/kg) increased the nuclear-Nrf2 level by $79.93 \%$ compared to $\mathrm{MCAO} / \mathrm{R}$ control rats. Those data indicated that CSA could activate the Nrf2 pathway in normal or t-BHP injured SH-SY5Y cells and MCAO/R rats, and the activation might relate to the regulation of Keap1.

Inhibition of Nrf2 pathway weakened the neuroprotective effect of CSA 
To explore whether activation of Nrf2 pathway contributed to the neuroprotection of CSA, Nrf2 pathway was suppressed with brusatol, a Nrf2 inhibitor (Cai, Liu, Han \& Yang, 2019). Fig. 7A indicated that brusatol inhibited the Nrf2 expression in a dose-dependent manner and the inhibitory rate was about $50 \%$ at concentration of $0.2 \mu \mathrm{M}$, so this concentration was used in the following experiments. Results showed that brusatol weakened the ability of CSA $(0.5 \mu \mathrm{M})$ to reduce the intracellular ROS level in t-BHP injured SH-SY5Y cells (Fig. 7B). In vivo studies further indicated that brusatol increased the infarct volume of CSAtreated MCAO/R rats, weakening the neuroprotective activity of CSA (Fig. 7C-D). Those data implied that inhibition of Nrf2 by brusatol attenuated the neuroprotective efficiency of CSA bothin vitro and in vivo . Hence, Nrf2 pathway might be critical for the antioxidant activity of CSA, being at least one of the primary targets for CSA-mediated neuroprotection.

\subsection{Inhibition of AMPK suppressed CSA induced Nrf2 activation}

Nrf2 activation was regulated by multiple kinases, including MAPKs, PI3K/AKT and AMPK, hence the upstream kinases that CSA might regulate to activate the Nrf2 pathway were further studied. Fig. 8A-F indicated that CSA enhanced the phosphorylation of Erk, p38 MAPK and AMPK in SH-SY5Y cells, while the phosphorylation of JNK and AKT was not affected by CSA. The kinases that related to CSA-mediated Nrf2 activation were further screened via applying a series of kinase inhibitors. Fig. 8N-O showed that all the inhibitors tested, including U0126 (Erk inhibitor), SB203580 (p38 MAPK \& AKT inhibitor), SP600125 (JNK inhibitor) and BML-275 (AMPK inhibitor) inhibited the CSA-induced Nrf2 nuclear translocation. Among them, the inhibitory rate of BML-275 was the highest, implying that AMPK might be the dominant kinase which CSA regulated to activate Nrf2 pathway. Hence, the AMPK activation in t-BHP injured SH-SY5Y cells and $\mathrm{MCAO} / \mathrm{R}$ rats was further tested. As indicated in Fig. 9A-B, CSA enhanced the phosphorylation of AMPK that was decreased by t-BHP or MCAO/R damages. To further study the role of AMPK in CSA-mediated antioxidation, intracellular ROS level in BML-275 treated SH-SY5Y cells were measured. Results showed that BML-275 enhanced the intracellular ROS level of CSA treated cells (Fig. 9C), that is, BML-275 weakened the antioxidant activity of CSA. Those data implied that AMPK was activated by CSA and AMPK/Nrf2 pathway might play essential role in CSA-mediated neuroprotection.

\section{Discussion}

So far only a few neuroprotective agents for ischemic stroke have been used clinically. Hence, it is necessary to develop more effective and novel agents for prevention or treatment of ischemic stroke. This study examined the neuroprotective activity of cajaninstilbene acid (CSA), an unique stilbenoid with a styryl group and the scaffold of aspirin. Aspirin possessed the free radical scavenging activity and was reported to activate the Nrf2/ARE pathway to protect the primary human melanocytes from $\mathrm{H}_{2} \mathrm{O}_{2}$ induced damages (Jian et al., 2016). It was also found to display neuroprotective effect in spinal cord injury model via activating the Nrf2/HO-1 signaling pathway (Wei et al., 2018). As for the styryl group, it might be an effective antioxidant group according to the studies of Hamdi et al., who reported that the coumarin derivatives possessing a styryl functional group exhibited much stronger antioxidant activity than all the other derivatives (Brahmachari, 2017). Our preliminary data with a group of simple stilbenoids also showed the importance of styryl group in neuroprotection against cerebral I/R damages. For those reasons, it was rational to suggest that CSA possessed good antioxidant activity and might exhibit neuroprotection in ischemic stroke. In this study, cerebral I/R injuries were established with two common models, that is, OGD/R model in SHSY5Y neuroblast cells and MCAO/R model in SD rats. Our study showed that CSA possessed significant neuroprotective activity, as evidenced by the reduced cell death and oxidative stress in OGD/R injured SH-SY5Y cells and decreased cerebral infarct volume and neurological deficits in MCAO/R rats. The most effective dose of CSA in MCAO/R rats was $5 \mathrm{mg} / \mathrm{kg}$ (i.p.) in this study. According to some previous studies, the frequently administrated dose of edaravone, a well-known neuroprotective drug, was $3-10 \mathrm{mg} / \mathrm{kg}$ (i.p. or i.v.) in the MCAO/R rat model (Srinivasan \& Sharma, 2012). Hence, the neuroprotective efficiency of CSA was relatively high, raising hope for its potential pharmacological use in ischemic stroke treatment. Besides, the neuroprotective activity of edaravone was primarily due to its antioxidant activity, which proved the effectiveness of antioxidant strategy for neuroprotection in clinical use (Fujiwara et al., 2016). CSA also 
exhibited antioxidant activity in ischemic stroke models, so we then tried to clarify its neuroprotective mechanism with the antioxidant pathway. As far as we know, this was the first study to comprehensively evaluate the neuroprotective activity of CSA against cerebral I/R injuries.

Cerebral I/R injuries enhanced the production of ROS, causing the imbalance between the generation and elimination, which led to inflammation and cell death via destroying proteins, DNAs and RNAs (Allen \& Bayraktutan, 2009). As mentioned above, we observed the antioxidant activity of CSA in the OGD/R model. To further explore the role of antioxidation in CSA mediated neuroprotection, SH-SY5Y cells was injured with t-BHP, a membrane permeable and relatively stable oxidant compound. t-BHP was commonly used to induced ROS production in cell-based studies (Fatemi et al., 2013). Our studies found that CSA could decrease ROS accumulation, enhance SOD activity, and lower the intracellular MDA content in t-BHP injured cells, and reduce the cell death, indicating that CSA exhibited neuroprotection via reducing I/R induced oxidative stress. Mitochondria were closely related to the intracellular redox homeostasis, in that, they were the main source of ROS, and meanwhile very susceptible to oxidative stress. Excessive ROS led to mitochondrial dysfunction, which then promoted the production of ROS, forming a vicious cycle (Yang, Mukda \& Chen, 2018). Hence, mitochondrial function was further measured in this study. Destruction of the energy metabolism was the most direct change in I/R damaged mitochondria. Normal mitochondrial membrane potential $\left(\Delta \Psi_{\mathrm{m}}\right)$ helped to maintain the proton gradient across the inner mitochondrial membrane, which was essential for ATP synthase to generate ATP (Zorova et al., 2018). Loss of $\Delta \Psi_{\mathrm{m}}$ was observed in t-BHP damaged cells, while CSA partially recovered the $\Delta \Psi_{m}$, which primarily showed the improvement of mitochondrial energy metabolism function. $\mathrm{Na}^{+}-\mathrm{K}^{+}$ATPase (NKA, sodium pump) and $\mathrm{Ca}^{2+}-\mathrm{Mg}^{2+}$ ATPase (CMA, calcium pump) translocated sodium and potassium or calcium across the plasma membrane using the energy from ATP hydrolysis to maintain the membrane potential and appropriate intracellular calcium level (Ji, Chauhan, Brown \& Chauhan, 2009). NKA and CMA could adjust their activity according to the rate of ATP production, so they could be used to evaluate the energy metabolism status (Bogdanova, Petrushanko, Hernansanz-Agustín \& Martínez-Ruiz, 2016). CSA was found to enhance the NKA and CMA activities at the presence of t-BHP or OGD/R injuries, further indicating that energy metabolism was improved after CSA treatment. Another important change of cerebral I/R damaged mitochondria was the formation of mitochondrial membrane permeability transition pore $(\mathrm{mPTP})$ in the inner mitochondrial membrane. mPTP led to the release of a series of pro-apoptotic factors, with cytochrome $\mathrm{c}$ to be the most representative one, to promote apoptosis of neurons (Andrabi, Ali, Tabassum, Parveen \& Parvez, 2019). CSA improved the mPTP, as evidenced by the reduced cytochrome c release after CSA treatment in t-BHP damaged SH-SY5Y cells. It could be concluded from these data that CSA lowered cellular oxidative stress and reduced the oxidative stress-induced mitochondrial dysfunction in SH-SY5Y cells.

Next, we investigated the underlying antioxidative mechanism of CSA. Nrf2 transcriptional factor, a primary host defense, was a critical important regulator of the intracellular redox status and functional integrity of mitochondria, especially under oxidative stress conditions (Dinkova-Kostova \& Abramov, 2015). Many reports have shown the important cytoprotective role of Nrf2 in cerebral I/R induced injuries and other neurodegenerative diseases. Some unique natural products have been reported to activate the Nrf2 pathway to decrease intracellular oxidative stress and attenuate mitochondrial dysfunction via inducing the expression of various antioxidant enzymes, such as HO-1 and NQO1 (Duan et al., 2019). Among them, sulforaphane and tert-butylhydroquinone exhibited good neuroprotection against cerebral I/R injuries and showed great potential in ischemic stroke treatment (Jiang et al., 2017). As for CSA, it was reported to activate the Nrf2/ARE pathway in HepG2 cells only (Liang et al., 2013). In this study, CSA was found to activate the Nrf2 pathway and induce the expression of HO-1 and NQO1 in normal or t-BHP damaged SH-SY5Y cells $(0.1-0.5 \mu \mathrm{M})$ as well as MCAO/R rats $(2.5-5 \mathrm{mg} / \mathrm{kg})$. Hence, Nrf2 pathway might contribute to CSAmediated neuroprotection. To illustrate this, brusatol was used to inhibit the Nrf2 pathway. Brusatol was a natural product isolated from Brucea javanica and has been proved to provoke rapid and transient Nrf2 depletion via the posttranscriptional mechanism (Cai, Liu, Han \& Yang, 2019). Ya et al. and Chen et al. indicated that brusatol worsened the brain injuries and Nrf2 activation in both transient focal cerebral I/R rat model and global cerebral I/R rat model (Chen et al., 2016; Ya et al., 2018). Therefore, it could 
be a reliable Nrf2 inhibitor. Our results showed that brusatol abolished the neuroprotection of CSA, as evidenced by the elevated ROS production in t-BHP injured SH-SY5Y cells and enhanced infarct volume in $\mathrm{MCAO} / \mathrm{R}$ rats. The activation of Nrf2 was related to Keap1, which bound to Nrf2 and inhibited its nuclear translocation. It was found that Keap1 modification by the electrophile accounted for Nrf2 activation $(\mathrm{Lv}$, Liu, Zhou, Tan, Deng \& Ci, 2017). We found that CSA decreased Keap1 expression in SH-SY5Y cells and $\mathrm{MCAO} / \mathrm{R}$ rats, which was associated with the increased Nrf2 nuclear translocation. Those data suggested that CSA might activate the Nrf2 pathway to reduce the oxidative stress and mitochondrial dysfunction caused by cerebral I/R injuries.

Given that Nrf2 was regulated by various signaling pathways, such as PI3K, MAPKs and AMPK, we then examined the effect of CSA on these pathways. Our result showed that CSA significantly enhanced the activation of Erk, p38 MAPK and AMPK, and the application of their specific inhibitors (U0126, SB203580, BML275) abolished CSA-induced Nrf2 nuclear translocation, with BML-275 showing the strongest inhibitory effect. Hence, CSA might regulate AMPK to further activate the Nrf2 pathway. AMPK was an important energy sensor that regulated the cellular energy homeostasis (Jiang et al., 2018). Some studies have indicated that AMPK activation was associated with the reduced oxidative stress, mitochondrial dysfunction and apoptosis, providing protective effect for various neurons, such as hippocampal neurons (Ronnett, Ramamurthy, Kleman, Landree \& Aja, 2009). In this study, a decrease of AMPK activation was observed under t-BHP induced oxidative stress and $\mathrm{MCAO} / \mathrm{R}$ conditions, while CSA reversed this decrease. The co-treatment with BML-275, an AMPK inhibitor weakened CSA's protective effect and enhanced the ROS formation. Hence, those data primarily indicated that CSA could enhance the activation of AMPK, which then promoted the Nrf2 activation. As for Erk and p38, suppressed Nrf2 activation was also observed with their corresponding inhibitors, so Erk and p38 might also contributed to CSA mediated Nrf2 activation.

In conclusion, our data demonstrated that CSA had significant neuroprotective activity in OGD/R or tBHP injured SH-SY5Y cells and MCAO/R rats. Those protective effects were exerted via activating the AMPK/Nrf2 pathway to enhance the expression of antioxidant enzymes, which then decreased the I/R induced oxidative stress and mitochondria dysfunction. This study provided scientific basis to use CSA for the prevention and treatment of ischemic stroke. However, more studies are still needed to further clarify the interaction mechanisms between AMPK and Nrf2 in the future.

\section{References}

Allen CL, Bayraktutan U (2009). Oxidative stress and its role in the pathogenesis of ischaemic stroke. Int J Stroke 4: 461-470.

Alscher RG, Erturk N, Heath LS (2002). Role of superoxide dismutases (SODs) in controlling oxidative stress in plants. J Exp Bot 53:1331-1341.

Andrabi SS, Ali M, Tabassum H, Parveen S, Parvez S (2019). Pramipexole prevents ischemic cell death via mitochondrial pathways in ischemic stroke. Dis Model Mech 12: dmm033860.

Bogdanova A, Petrushanko IY, Hernansanz-Agustín P, Martínez-Ruiz A (2016). "Oxygen sensing" by $\mathrm{Na}^{+}$, $\mathrm{K}^{+}$-ATPase: these miraculous thiols. Front Physiol 7: 314 .

Brahmachari G (2017). Discovery and development of neuroprotective agents from natural products. Amsterdam: Elsevier.

Cai SJ, Liu Y, Han S, Yang C (2019). Brusatol, an NRF2 inhibitor for future cancer therapeutic. Cell Biosci 9: 45 .

Chang RC, Ho YS (2019). Introductory chapter: Concept of neuroprotection-A new perspective in neuroprotection. London: IntechOpen.

Chen B, Cao H, Chen L, Yang X, Tian X, Li R, et al. (2016). Rifampicin attenuated global cerebral ischemia injury via activating the nuclear factor erythroid 2-related factor pathway. Front Cell Neurosci 10: 273. 
de Vries HE, Witte M, Hondius D, Rozemuller AJ, Drukarch B, Hoozemans J, et al. (2008). Nrf2-induced antioxidant protection: a promising target to counteract ROS-mediated damage in neurodegenerative disease? Free Radic Biol Med 45: 1375-1383.

Dinkova-Kostova AT, Abramov AY (2015). The emerging role of Nrf2 in mitochondrial function. Free Radic Biol Med 88: 179-188.

Doyle KP, Simon RP, Stenzel-Poore MP (2008). Mechanisms of ischemic brain damage. Neuropharmacology 55: 310-318.

Duan J, Cui J, Yang Z, Guo C, Cao J, Xi M, et al. (2019). Neuroprotective effect of Apelin 13 on ischemic stroke by activating AMPK/GSK-3 $\beta /$ Nrf2 signaling. J Neuroinflammation 16: 24.

Fatemi N, Sanati M, Jamali Zavarehei M, Ayat H, Esmaeili V, Golkar-Narenji A, et al. (2013). Effect of tertiary-butyl hydroperoxide (TBHP)-induced oxidative stress on mice sperm quality and testis histopathology. Andrologia 45: 232-239.

Feng J, Chen X, Lu S, Li W, Yang D, Su W, et al. (2018). Naringin attenuates cerebral ischemia-reperfusion injury through inhibiting peroxynitrite-mediated mitophagy activation. Mol Neurobiol 55:9029-9042.

Fujiwara N, Som AT, Pham LD, Lee BJ, Mandeville ET, Lo EH, et al.(2016). A free radical scavenger edaravone suppresses systemic inflammatory responses in a rat transient focal ischemia model. Neurosci Lett 633: 7-13.

Huang M, Lin J, Lu K, Xu H, Geng Z, Sun P, et al. (2016). Anti-inflammatory effects of cajaninstilbene acid and its derivatives. J Agric Food Chem 64: 2893-2900.

Ji L, Chauhan A, Brown WT, Chauhan V (2009). Increased activities of $\mathrm{Na}^{+} / \mathrm{K}^{+}-\mathrm{ATPase}$ and $\mathrm{Ca}^{2+} / \mathrm{Mg}^{2+}$ ATPase in the frontal cortex and cerebellum of autistic individuals. Life Sci 85:788-793.

Ji X, Chen J, Zheng G, Huang M, Zhang L, Yi H, et al. (2016). Design and synthesis of cajanine analogues against hepatitis $\mathrm{C}$ virus through down-regulating host chondroitin sulfate $\mathrm{N}$-acetylgalactosaminyltransferase 1. J Med Chem 59:10268-10284.

Jian Z, Tang L, Yi X, Liu B, Zhang Q, Zhu G, et al. (2016). Aspirin induces Nrf2-mediated transcriptional activation of haem oxygenase-1 in protection of human melanocytes from $\mathrm{H} 2 \mathrm{O} 2$-induced oxidative stress. J Cell Mol Med 20: 1307-1318.

Jiang B, Liu Y, Le L, Li Z, Si J, Liu X, et al. (2014). Cajaninstilbene acid prevents corticosterone-induced apoptosis in PC12 cells by inhibiting the mitochondrial apoptotic pathway. Cell Physiol Biochem 34: 10151026.

Jiang S, Deng C, Lv J, Fan C, Hu W, Di S, et al. (2017). Nrf2 weaves an elaborate network of neuroprotection against stroke. Mol Neurobiol 54: 1440-1455.

Jiang S, Li T, Ji T, Yi W, Yang Z, Wang S, et al. (2018). AMPK: potential therapeutic target for ischemic stroke. Theranostics 8: 4535.

Joo MS, Kim WD, Lee KY, Kim JH, Koo JH, Kim SG (2016). AMPK facilitates nuclear accumulation of Nrf2 by phosphorylating at serine 550. Mol Cell Biol 36: 1931-1942.

Kunz A, Dirnagl U, Mergenthaler P (2010). Acute pathophysiological processes after ischaemic and traumatic brain injury. Best Pract Res Clin Anaesthesiol 24: 495-509.

Liang L, Luo M, Fu Y, Zu Y, Wang W, Gu C, et al. (2013). Cajaninstilbene acid (CSA) exerts cytoprotective effects against oxidative stress through the Nrf2-dependent antioxidant pathway. Toxicol Lett 219: 254-261.

Liu X, Zhou D, Xie T, Malik TH, Lu C, Li H, et al. (2017). Nrf2, a potential therapeutic target against oxidative stress in corneal diseases. Oxid Med Cell Longev 2017: 2326178. 
Lv H, Liu Q, Zhou J, Tan G, Deng X, Ci X (2017). Daphnetin-mediated Nrf2 antioxidant signaling pathways ameliorate tert-butyl hydroperoxide (t-BHP)-induced mitochondrial dysfunction and cell death. Free Radic Biol Med 106: 38-52.

Mao L, Jia J, Zhou X, Xiao Y, Wang Y, Mao X, et al. (2013). Delayed administration of a PTEN inhibitor BPV improves functional recovery after experimental stroke. Neuroscience 231: 272-281.

Qi Z, Ci X, Huang J, Liu Q, Yu Q, Zhou J, et al. (2017). Asiatic acid enhances Nrf2 signaling to protect HepG2 cells from oxidative damage through Akt and ERK activation. Biomed Pharmacother 88:252-259.

Ronnett GV, Ramamurthy S, Kleman AM, Landree LE, Aja S (2009). AMPK in the brain: its roles in energy balance and neuroprotection. J Neurochem 109: 17-23.

Sanderson TH, Reynolds CA, Kumar R, Przyklenk K, Hüttemann M (2013). Molecular mechanisms of ischemia-reperfusion injury in brain: pivotal role of the mitochondrial membrane potential in reactive oxygen species generation. Mol Neurobiol 47: 9-23.

Simion A, Jurcau A (2019). The role of antioxidant treatment in acute ischemic stroke: past, present and future. Neurol Res Surg 2:1-7.

Srinivasan K, Sharma SS (2012). Edaravone offers neuroprotection in a diabetic stroke model via inhibition of endoplasmic reticulum stress. Basic Clin Pharmacol Toxicol 110: 133-140.

Sun Y, Liu Y, He W, Wang C, Tickner J, Kuek V, et al. (2019). Cajaninstilbene acid inhibits osteoporosis through suppressing osteoclast formation and RANKL-induced signaling pathways. J Cell Physiol 234: 11792-11804.

Syed R, Wu Y (2018). A review article on health benefits of Pigeon pea (Cajanus cajan (L.) Millsp). Nutr Res 2: 15 .

Wang Y, Huang Y, Xu Y, Ruan W, Wang H, Zhang Y, et al. (2018). A dual AMPK/Nrf2 activator reduces brain inflammation after stroke by enhancing microglia M2 polarization. Antioxid Redox Signal 28:141-163.

Watanabe K, Tanaka M, Yuki S, Hirai M, Yamamoto Y (2018). How is edaravone effective against acute ischemic stroke and amyotrophic lateral sclerosis? J Clin Biochem Nutr 62: 20-38.

Wei W, Shurui C, Zipeng Z, Hongliang D, Hongyu W, Yuanlong L, et al. (2018). Aspirin suppresses neuronal apoptosis, reduces tissue inflammation, and restrains astrocyte activation by activating the Nrf2/HO-1 signaling pathway. Neuroreport 29: 524-531.

Wu C, Chen J, Yang R, Duan F, Li S, Chen X (2019). Mitochondrial protective effect of neferine through the modulation of nuclear factor erythroid 2-related factor 2 signalling in ischaemic stroke. Br J Pharmacol 176: 400-415.

Ya B, Liu Q, Li H, Cheng H, Yu T, Chen L, et al. (2018). Uric acid protects against focal cerebral ischemia/reperfusion-induced oxidative stress via activating Nrf2 and regulating neurotrophic factor expression. Oxid Med Cell Longev 2018: 6069150.

Yang J, Mukda S, Chen S (2018). Diverse roles of mitochondria in ischemic stroke. Redox Biol 16: 263-275.

Zorova LD, Popkov VA, Plotnikov EY, Silachev DN, Pevzner IB, Jankauskas SS, et al. (2018). Mitochondrial membrane potential. Anal Biochem 552: 50-59.

\section{Figure legends}

\section{Fig.1 Chemical structure of CSA.}

Fig. 2 Neuroprotective effect of CSA against cerebral I/R and t-BHP induced injuries. (A) SH-SY5Y cells were subjected to OGD/R damages after $24 \mathrm{~h}$ CSA pretreatment. Cell viability was then measured with the CCK-8 method. (B-C) SH-SY5Y cells were pretreated with CSA for $24 \mathrm{~h}$, and then 
damaged with $100 \mu \mathrm{M}$ t-BHP. (B) Cell viability was measured with the CCK-8 method. (C) LDH level in the cultural supernatant was tested using a pierce LDH cytotoxicity assay kit. (D-F) MCAO/R rats ( $\mathrm{n}=10$ ) were intraperitoneally injected with CSA at the onset of reperfusion. After $24 \mathrm{~h}$, (D) Brain tissues were stained with TTC method and photographed. (E) Infarct volume was calculated with the formula: (right hemisphere size - red part size of left hemisphere) / (right hemisphere size $\times 2$ ) $\times 100 \%$. (F) Neurological deficits were scored with the mNSS scale. All results were expressed as means \pm SD of at least three independent experiments. ${ }^{\#} P<0.05$; ${ }^{\# \#} P<0.01$ versus control or sham control group; ${ }^{*} P<0.05 ;{ }^{* *} P<$ 0.01 versus $\mathrm{OGD} / \mathrm{R}$ or $\mathrm{MCAO} / \mathrm{R}$ control group.

Fig. 3 Antioxidant activity of CSA in SH-SY5Y cells.(A-C) SH-SY5Y cells were pretreated with CSA for $24 \mathrm{~h}$, and then damaged with $100 \mu \mathrm{M}$ t-BHP. (A) Intracellular ROS level was measured with DCFH-DA dye after $4 \mathrm{~h}$. (B-C) MDA content and SOD activity were measured with corresponding kits after $12 \mathrm{~h}$. (DF) SH-SY5Y cells were subjected to $8 \mathrm{~h}$ OGD damages after $24 \mathrm{~h}$ CSA pretreatment. (D) Intracellular ROS level was measured with DCFH-DA dye after reperfusion for $2 \mathrm{~h}$. (E-F) MDA content and SOD activity were measured after reperfusion for $16 \mathrm{~h}$. All results were expressed as means $\pm \mathrm{SD}$ of at least three independent experiments. ${ }^{*} P<0.05 ;{ }^{\# \#} P<0.01$ versus control or sham control group; ${ }^{*} P<0.05 ;{ }^{* *} P<0.01$ versus $\mathrm{OGD} / \mathrm{R}$ or $\mathrm{MCAO} / \mathrm{R}$ control group.

Fig. 4 CSA improved mitochondrial function of t-BHP or OGD/R injured SH-SY5Y cells. (A-F) SH-SY5Y cells were pretreated with CSA for $24 \mathrm{~h}$ and then damaged with $100 \mu \mathrm{M}$ t-BHP. (A-C) $\Delta \Psi_{\mathrm{m}}$ was measured with JC-1 dye by flow cytometry after $4 \mathrm{~h}$. (A) Dot-plots of the JC- 1 stained cells. (B) Analysis of the percentage of cells with decrease $\Delta \Psi_{\mathrm{m}}$ (depolarized cells) from the dot-plots. (C) Quantitative analysis of the ratio of red/green signal. (D) Cytosolic fraction was separated, and cytosolic cytochrome c level was measured by Western blotting. $\beta$-actin was used as the internal control. Western blotting bands were semiquantitatively analyzed using Image $J$ software. (E-F) $\mathrm{Na}^{+}-\mathrm{K}^{+}$ATPase and $\mathrm{Ca}^{2+}-\mathrm{Mg}^{2+}$ ATPase activities were detected with a commercial kit. (G-H) Cells were subjected to OGD/R after $24 \mathrm{~h}$ CSA $(0.5 \mu \mathrm{M})$ pretreatment. $\mathrm{Na}^{+}-\mathrm{K}^{+}$ATPase and $\mathrm{Ca}^{2+}-\mathrm{Mg}^{2+}$ ATPase activities were then detected with a commercial kit. All results were expressed as means $\pm \mathrm{SD}$ of at least three independent experiments. $\#$ \# $<0.01$ versus control group; ${ }^{*} P<0.05 ;{ }^{* *} P<0.01$ versus OGD/R or t-BHP group.

Fig. 5 CSA activated Nrf2 pathway in SH-SY5Y cells. (A-F) SH-SY5Y cells were pretreated with CSA for 24 h. (A-D) Proteins were collected and protein levels of Nrf2, HO-1 and NQO1 were detected with Western blotting. $\beta$-actin was used as an internal control. (E-F) Nuclear fractions were separated with a commercial kit and nuclear Nrf2 level was measured by Western blotting. Histone H3 (HH3) was used as an internal control. (G-L) SH-SY5Y cells were pretreated with CSA for $24 \mathrm{~h}$ and then damaged with $100 \mu \mathrm{M}$ t-BHP. (G-K) Proteins were collected and protein levels of Nrf2, HO-1, NQO1 and Keap1 were detected with Western blotting. $\beta$-actin was used as an internal control. (L) Nuclear fractions were separated and nuclear Nrf2 level was measured by Western blotting. HH3 was used as an internal control. All Western blotting bands were semi-quantitatively analyzed using Image $\mathrm{J}$ software. All results were expressed as means $\pm \mathrm{SD}$ of at least three independent experiments. ${ }^{\#} P<0.01$ versus control group; ${ }^{*} P<0.05 ;{ }^{* *} P<0.01$ versus t-BHP group.

Fig. 6 CSA activated Nrf2 pathway in MCAO/R rats. (A-F) MCAO/R rats were intraperitoneally injected with CSA at the onset of reperfusion. After $24 \mathrm{~h}$, (A-E) Proteins of rat brains were collected and protein levels of Nrf2, HO-1, NQO1 and Keap1 were detected with Western blotting. $\beta$-actin was used as an internal control. (F) Nuclear fractions of rat brains were separated and nuclear Nrf2 level was measured by Western blotting. HH3 was used as an internal control. All Western blotting bands were semi-quantitatively analyzed using Image $\mathrm{J}$ software. All results were expressed as means $\pm \mathrm{SD}$ of at least three independent experiments. ${ }^{\# \#} P<0.01$ versus control group; ${ }^{* *} P<0.01$ versus $\mathrm{MCAO} / \mathrm{R}$ group.

Fig. 7 Inhibition of Nrf2 weakened the neuroprotective effect of CSA. (A) SH-SY5Y cells were treated with brusatol for $24 \mathrm{~h}$, and then proteins were collected. Nrf2 protein level was measured with Western blotting. $\beta$-actin was used as an internal control. Western blotting bands were semi-quantitatively analyzed using Image J software. (B) SH-SY5Y cells were pretreated with CSA (0.5 $\mu \mathrm{M})$ or brusatol for 24 
$\mathrm{h}$ and then damaged with $100 \mu \mathrm{M}$ t-BHP. After $4 \mathrm{~h}$, intracellular ROS level was measured with DCFH-DA dye. (C-D) MCAO/R rats were intraperitoneally injected with CSA or brusatol at the onset of reperfusion. After $24 \mathrm{~h},(\mathrm{C})$ Brain tissues were stained with TTC method and photographed. (D) Infarct volume was calculated with the formula: (right hemisphere size - red part size of left hemisphere) / (right hemisphere size $\times 2) \times 100 \%$. All results were expressed as means \pm SD of at least three independent experiments. \#\# $P$ $<0.01$ versus control or sham control group; ${ }^{* *} P<0.01$ versus $\mathrm{t}-\mathrm{BHP}$ or $\mathrm{MCAO} / \mathrm{R}$ group; \&\& $P<0.01$ versus $\mathrm{t}-\mathrm{BHP}+\mathrm{CSA}$ or MCAO/R + CSA group.

Fig. 8 Screening of the upstream kinases that CSA regulated to activate Nrf2. (A-F) SH-SY5Y cells were pretreated with CSA for $24 \mathrm{~h}$. Proteins were collected, and protein levels of the kinases were detected with Western blotting. (G-M) SH-SY5Y cells were treated with different kinase inhibitors for $24 \mathrm{~h}$. Proteins were collected, and protein levels of the corresponding kinases were detected with Western blotting. (N-O) SH-SY5Y cells were pretreated with CSA $(0.5 \mu \mathrm{M})$ and different kinase inhibitors for $24 \mathrm{~h}$. Nuclear fractions were separated with a commercial kit and nuclear Nrf2 level was measured by Western blotting. HH3 was used as an internal control. All Western blotting bands were semi-quantitatively analyzed using Image $\mathrm{J}$ software. All results were expressed as means $\pm \mathrm{SD}$ of at least three independent experiments. ${ }^{\#} P$ $<0.05 ; \# \# P 0.01$ versus control group; ${ }^{*} P<0.05 ;{ }^{* *} P<0.01$ versus CSA group.

Fig. 9 Inhibition of AMPK weakened the antioxidative activity of CSA. (A) SH-SY5Y cells were pretreated with CSA and then damaged with $100 \mu \mathrm{M}$ t-BHP. Proteins were collected, and protein levels of p-AMPK and AMPK were detected with Western blotting. (B) MCAO/R rats were intraperitoneally injected with CSA at the onset of reperfusion. After $24 \mathrm{~h}$, proteins of rat brains were collected, and protein levels of p-AMPK and AMPK were detected with Western blotting. (C) SH-SY5Y cells were pretreated with CSA $(0.5 \mu \mathrm{M})$ and BML-275 and then damaged with $100 \mu \mathrm{M}$ t-BHP. After $4 \mathrm{~h}$, intracellular ROS level was measured with DCFH-DA dye. All Western blotting bands were semi-quantitatively analyzed using Image $\mathrm{J}$ software. All results were expressed as means $\pm \mathrm{SD}$ of at least three independent experiments. $\# \# P<0.01$ versus control group; ${ }^{* *} P<0.01$ versus $\mathrm{t}-\mathrm{BHP}$ or $\mathrm{MCAO} / \mathrm{R}$ group; ${ }^{\&} \& P<0.01$ versus $\mathrm{t}-\mathrm{BHP}+\mathrm{CSA}$ group.

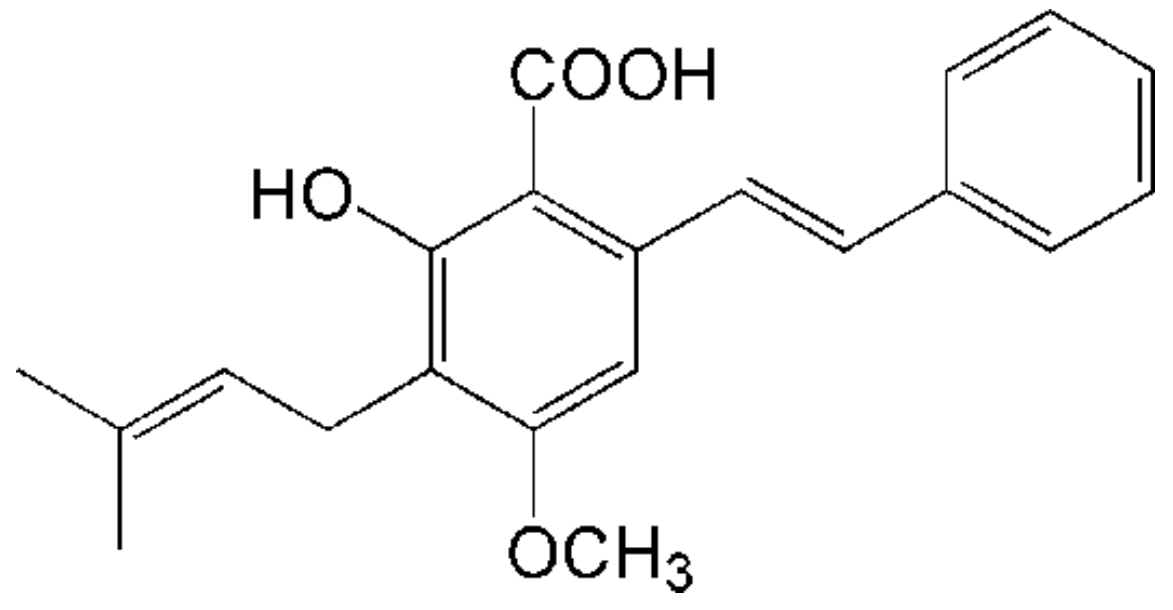



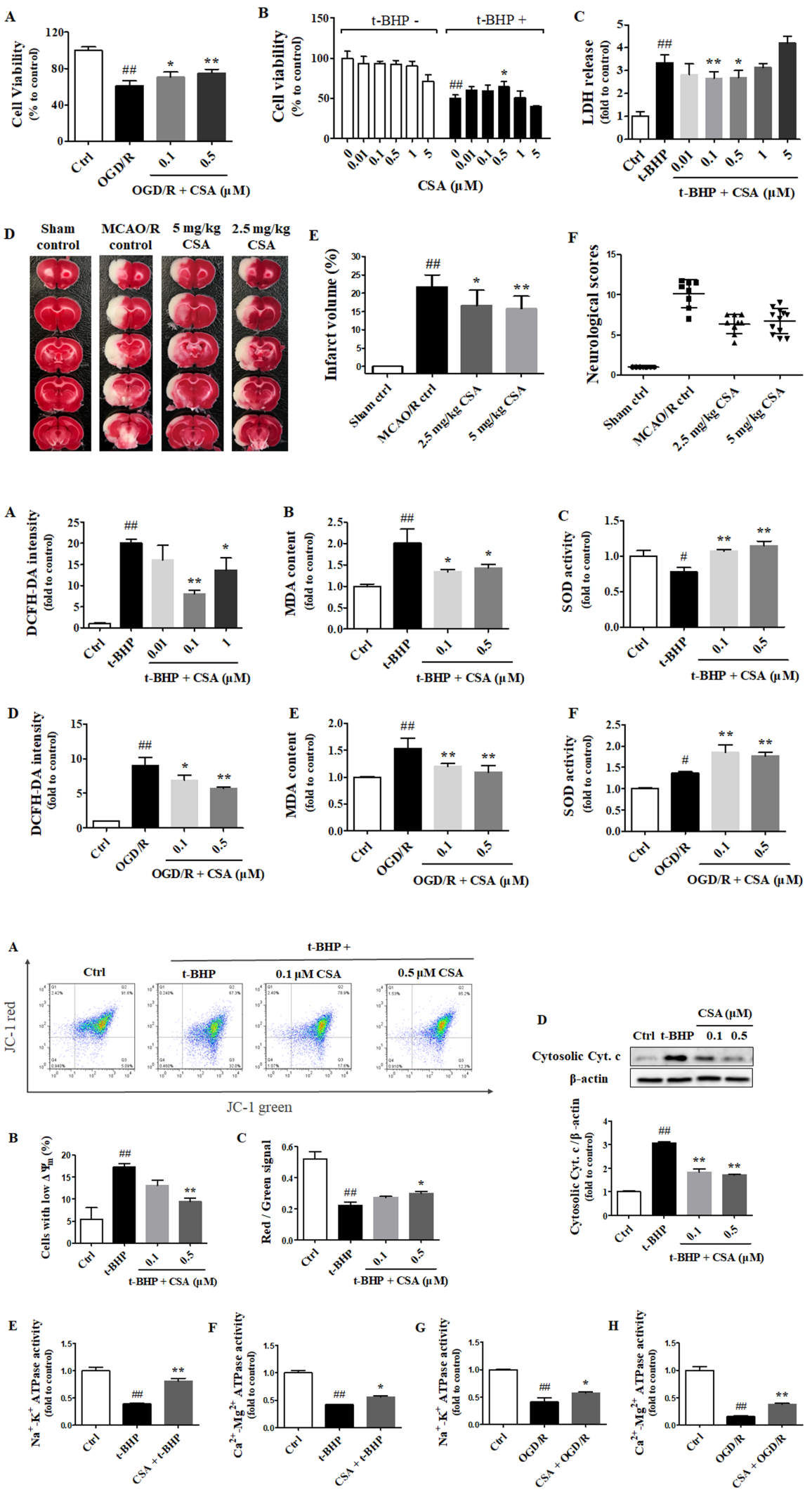


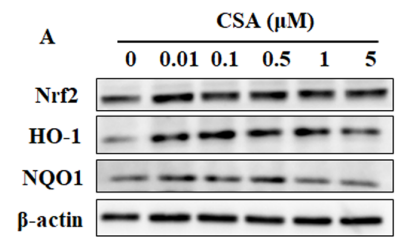

D

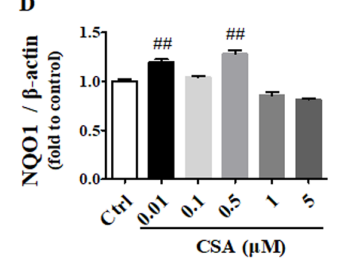

G

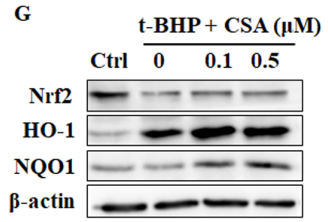

$\mathbf{J}$

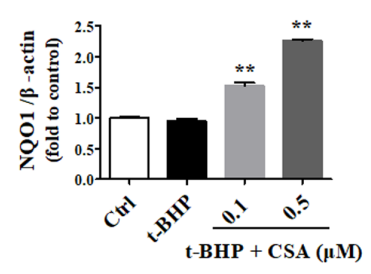

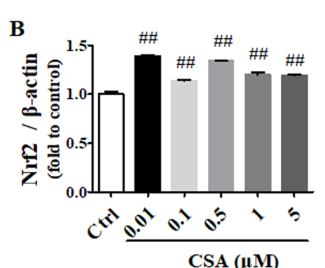

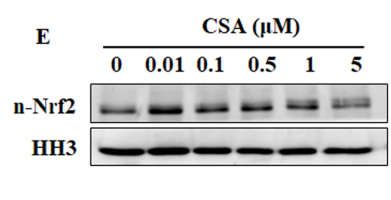

H

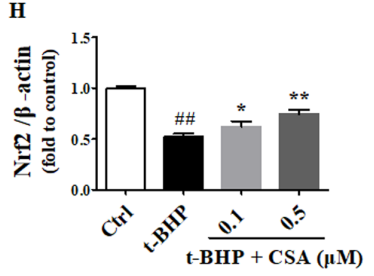

K

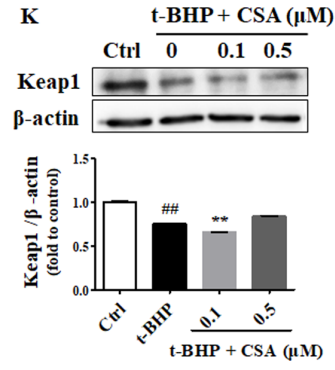

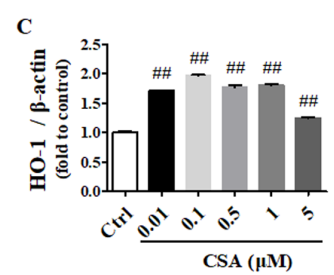

F
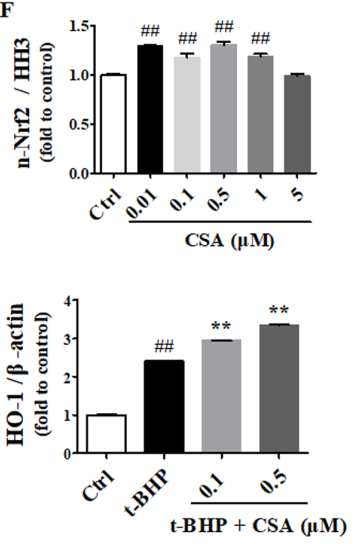

L t-BHP + CSA $(\mu \mathrm{M})$

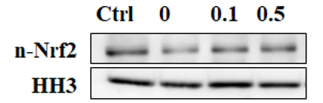

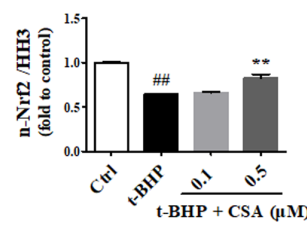

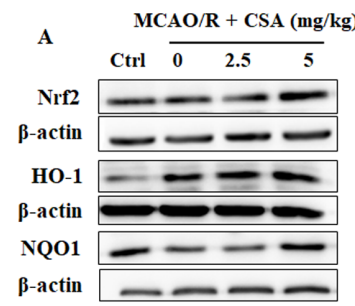
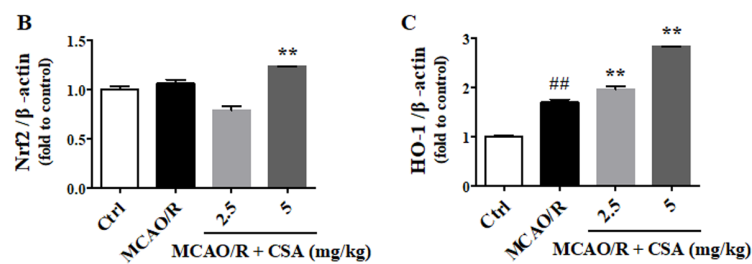

D
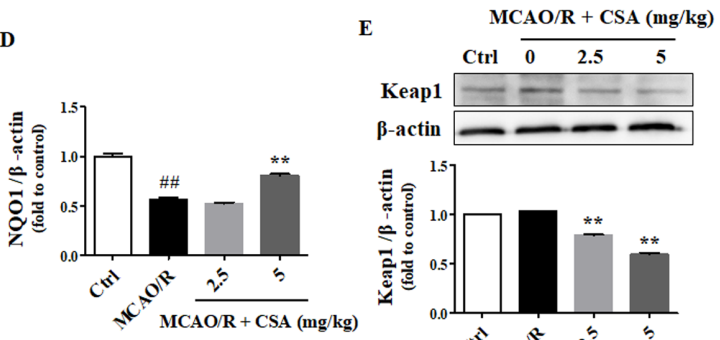

है
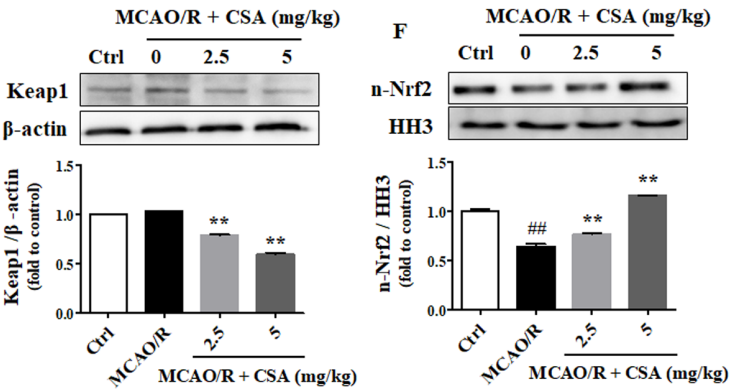

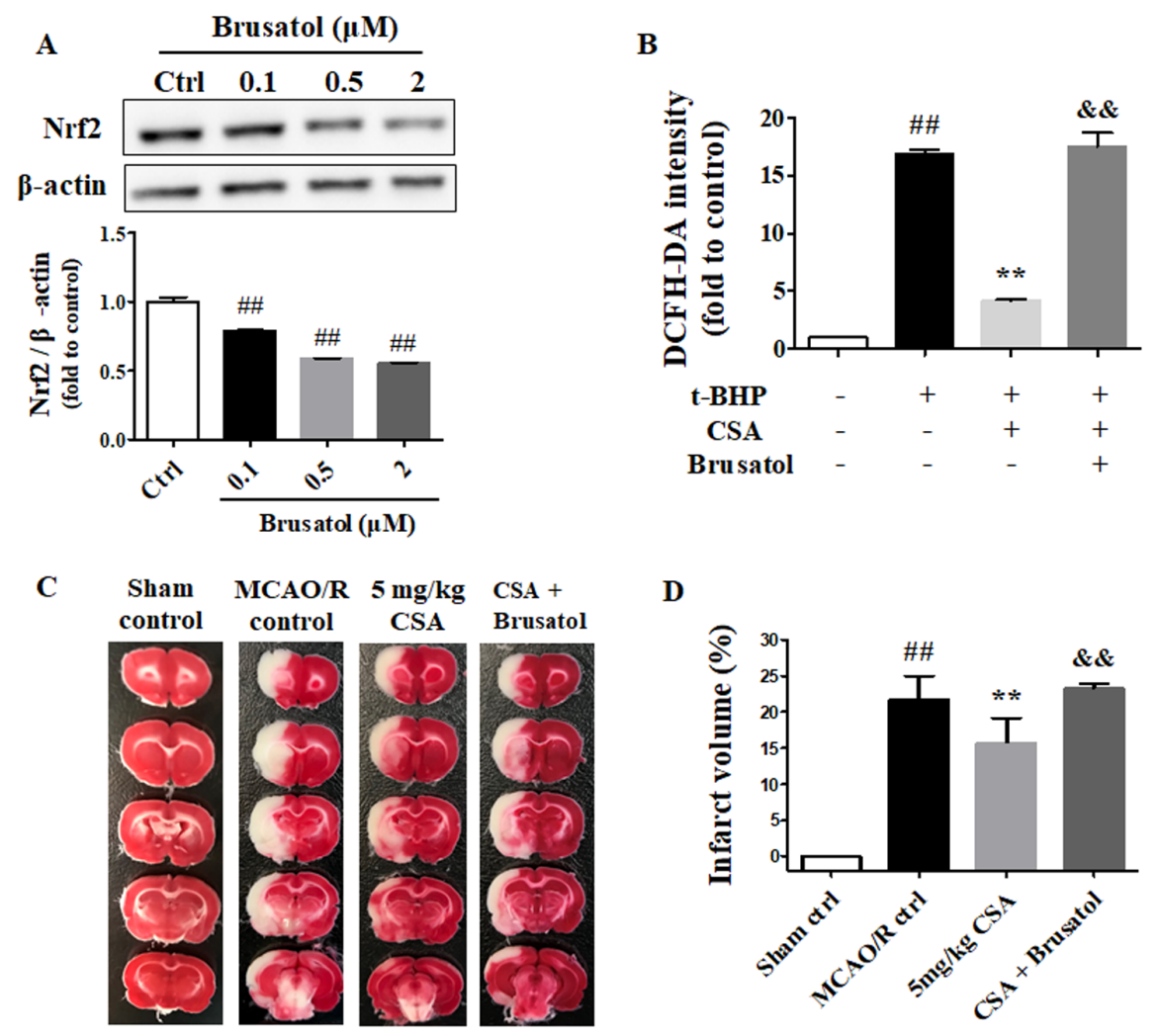

D

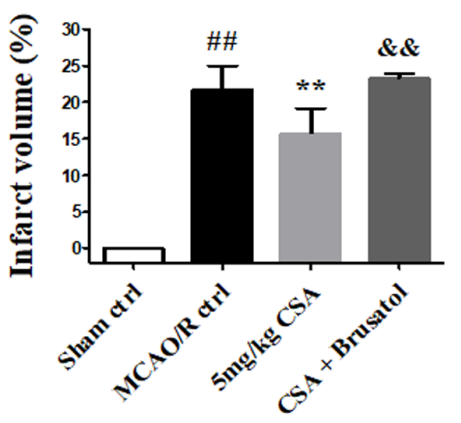



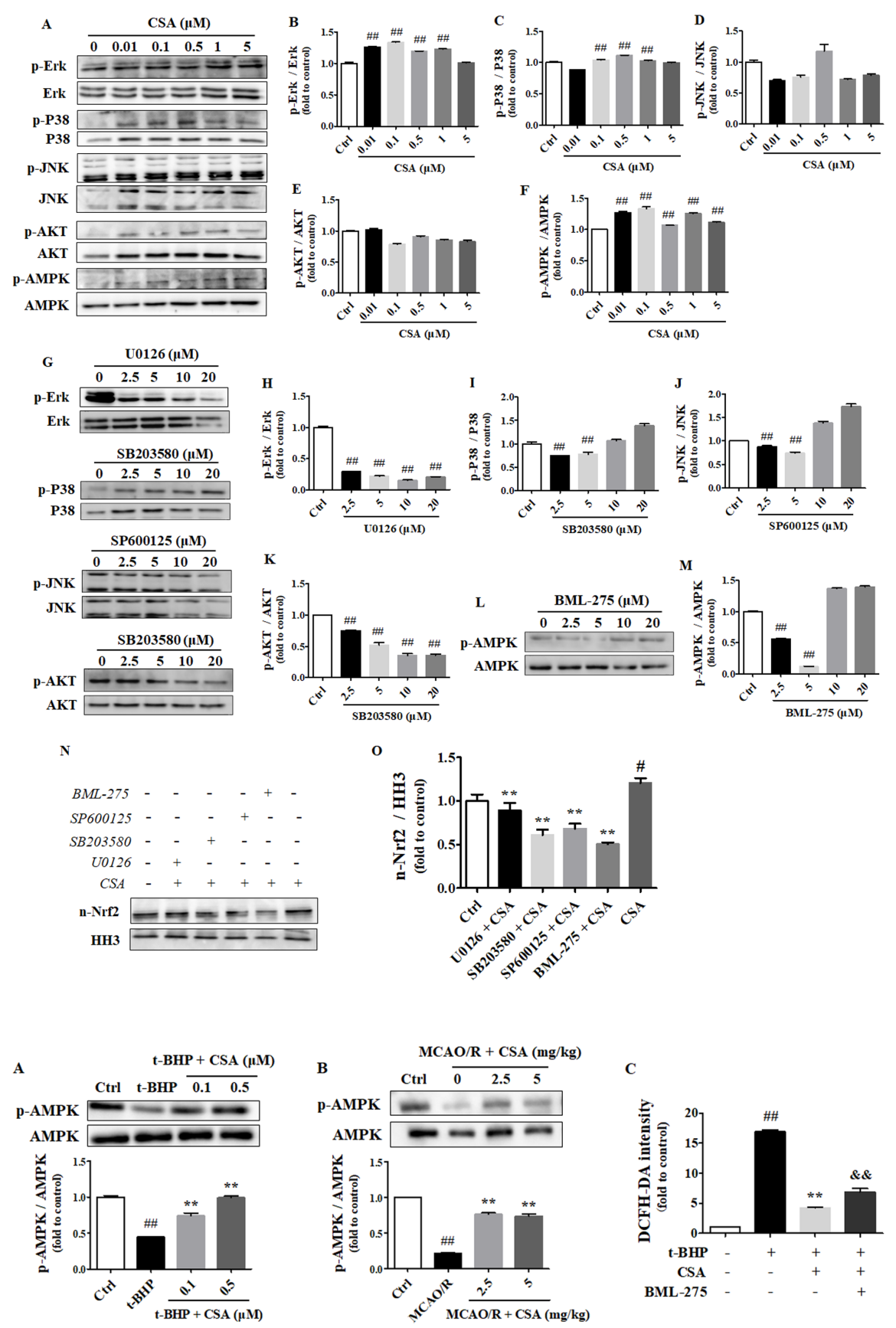